\title{
Fog Juice Poisoning
}

\author{
ISLAM FMM, ${ }^{1}$ SAHA AK, ${ }^{2}$ MANNAN MA ${ }^{3}$ HASSAN TU, ${ }^{4}$ RAHMAN M, ${ }^{5}$ AHMED F ${ }^{6}$, SAHA BN, ${ }^{6}$ ROY H, ${ }^{6}$ RAHMAN M, ${ }^{6}$ \\ CHOWDHURY NS 7
}

\begin{abstract}
:
A 19 yrs old male was admitted with upper abdominal pain and vomiting following ingestion of a fluid he thought to be beer. After repeated inquiry it was found that the clear fluid was "Fog Juice", used to produce artificial "Fog" or Smoke on the stage. After 36 hours of ingestion the patient developed severe respiratory distress \& itching. Investigation reports confirmed Acute Renal Failure \& Hyperkalaemia. He was sent to Nephrology Unit for dialysis, but the poor victim expired there about 48 hours after ingestion.
\end{abstract}

Keywords: Fog Juice, Glycols

\section{Introduction:}

Fog juice is a fluid that is used to make artificial fog or cloud on the stage during concert or drama to make a different divinely atmosphere. In most smoke machine a "Glycol" based fluid is pumped into a heated chamber. The fluid evaporates very quickly so vapour exits under pressure and upon contact with the cool air it forms a dense cloud that is very similar to real fog. Commonly used ingredients to make fog juice include: 1. Distilled water. 2. Glycerine. 3. Diethylene glycol, 4. Dipropylene glycol, 5. Propylene glycol, 6. Triethylene glycol, etc. There are numerous kinds of glycol, most of them are poisonous but some are safe and are used in food and medicine industry. Just a little change in name can make a big difference and using wrong chemicals can kill people.

\section{Case Report:}

A 19 yrs old male was admitted in Medicine Unit- IV of Sir Salimullah Medical College and Mitford Hospital (SSMC \& $\mathrm{MH}$ ), on $25^{\text {th }}$ December 2010 at 12:30 AM, with the complaints of pain in the upper abdomen and vomiting for couple of times within one hour following ingestion of about $100 \mathrm{ml}$ of

1. Assoc. Professor, Dept. of Medicine, Sir Salimullah Medical College \& Hospital, Dhaka

2. Professor, Dept. of Medicine, Sir Salimullah Medical College \& Hospital, Dhaka

3. Asst. Professor, Dept. of Medicine, Sir Salimullah Medical College \& Hospital, Dhaka

4. Registrar, Dept. of Medicine, Sir Salimullah Medical College \& Hospital, Dhaka

5. Assistant registrar, Dept. of Medicine, Sir Salimullah Medical College \& Hospital, Dhaka

6. Medical Officer, Dept. of Medicine, Sir Salimullah Medical College \& Hospital, Dhaka

7. Assistant professor, Dept. of Biochemistry, National Institute of Diseases of Chest and Hospital. Mohakhali. Dhaka.

Correspondence: Dr. FM Mofakharul Islam. Associate professor of Medicine, Sir Salimullah Medical College \& Mitford Hospital, Dhaka. House No: 12/A (704), Eskaton Garden Road, Ramna, Dhaka-1000. E-mail:mofakharulislam@yahoo.com.Mobile: 01711812191 a colorless fluid, he thought to be beer. Stomach wash was given in the emergency department. Patient was conscious $\&$ vital signs were normal. He was kept nothing by mouth with intravenous fluid, Thiamine \& Ranitidine. The following day was uneventful.

On the next ( $26^{\text {th }}$ December) morning about 34 hours later the patient was found to be dyspnoeic which aggravated gradually over time, he also developed intense itching. He was conscious but anxious and said that he has not passed any urine for last 16 hours. His respiratory rate was rapid, pulse 100/min, BP was 150/90 mm of Hg. His chest and abdomen examination revealed no abnormality. A bed side ECG showed tall peaked "T" wave. Patient's blood sample showed: Urea- $136 \mathrm{mg} / \mathrm{dl}$, Creatinine $-8 \mathrm{mg} / \mathrm{dl}, \mathrm{Na}^{+}-126 \mathrm{mmol}$ $/ \mathrm{L}, \mathrm{K}^{+} 5.5 \mathrm{mmol} / \mathrm{L}, \mathrm{Cl}^{-}-97 \mathrm{mmol} / \mathrm{L}, \mathrm{HCO}_{3}{ }^{-}-18 \mathrm{mmol} / \mathrm{L}$. After repeated questions the patient and his attendant said that that the clear fluid he took was fog juice. Patient was urgently transferred to Nephrology Department. Intermittent Peritoneal Dialysis was initiated but patient died at that night (about 48 hours after ingestion of the acquiesced fluid).

\section{Discussion:}

There are numerous types of glycols. Examples of some glycols in common use are mentioned below:

Ethylene glycol (Ethane-1,2-diol) is an organic compound, in its pure form, it is an odorless, colorless, syrupy, sweettasting liquid. Ethylene glycol is not to be confused with diethylene glycol, a heavier ether diol, or with polyethylene glycol, a nontoxic polyether polymer. The major uses of ethylene glycol are to make PET melt, automotive antifreeze and a precursor to polymers. Minor uses of ethylene glycol include the manufacture of capacitors, shoe polish, some inks and dyes. Ethylene glycol is commonly used as a preservative for biological as a safer alternative to formaldehyde. It can also be used in killing jars. ${ }^{1}$ Ethylene glycol is moderately toxic. ${ }^{2}$ Upon ingestion, ethylene glycol is oxidized to glycolic acid which is, in turn, oxidized to oxalic 
acid, which is toxic. It and its toxic byproducts first affect the central nervous system, then the heart, and finally the kidneys. Ingestion of sufficient amounts can be fatal if untreated. ${ }^{3}$

Diethylene glycol (DEG) ${ }^{4}$ is an organic compound. It is a colorless, practically odorless, and hygroscopic liquid with a sweetish taste. It is miscible in water, alcohol, ether, acetone and ethylene glycol. DEG is a widely used solvent. Diethylene glycol is used in the manufacture of unsaturated polyester resins, polyurethanes and plasticizers It is a humectant for tobacco, cork, printing ink, and glue. It is also a component in brake fluid, lubricants, wallpaper strippers, artificial fog solutions, and heating/cooking fuel. A dilute solution of diethylene glycol can also be used as a coolant; however, ethylene glycol is much more commonly used. It is very poisonous. Some authors suggest that minimum toxic dose is estimated at $0.14 \mathrm{mg} / \mathrm{kg}$ of body weight and lethal dose between 1 and $1.63 \mathrm{~g} / \mathrm{kg}$ of body weight, Because of its adverse effects on humans, diethylene glycol is not allowed for use in food and drugs. Its use in adulterated consumer products has resulted in numerous epidemics of poisoning since the early 20th century.

Triethylene glycol ${ }^{5}$ is a colorless odorless viscous liquid. It is used as a plasticizer for vinyl. Because of its exceptionally low toxicity and low odor combined with its antimicrobial properties it is used in air sanitizer products. Triethylene glycols are also used as liquid desiccants for natural gas and in air conditioning systems. It is an additive for hydraulic fluids and brake fluids .

Polyethylene glycol (PEG) $)^{6,7}$ is a polyether compound; it has a low toxicity and is used in a variety of products, from industrial manufacturing to medicine. PEG is used as an excipient in pharmaceutical products. Lower-molecularweight variants are used as solvents in oral liquids and soft capsules, whereas solid variants are used as ointment bases, tablet binders, film coatings, and lubricants. It is the basis of many skin creams, and sexual lubricants, laxatives and lubricant eye drops . Polyethylene glycol is the primary component in a type of antifreeze solution used in automobiles and boats as a low-toxicity alternative to the traditional highly poisonous ethylene glycol solutions used in standard antifreeze products.

Propylene glycol, ${ }^{8}$ also called 1, 2-propanediol or propane1, 2-diol, is an organic compound. It is a colorless, nearly odorless, clear, viscous liquid with a faintly sweet taste, hygroscopic and miscible with water, acetone, and chloroform. It is used as a solvent in many pharmaceuticals, including oral, injectable and topical formulations. As a moisturizer in medicines, cosmetics, food, toothpaste, shampoo, mouth wash, hair care and tobacco products. In hand sanitizers, antibacterial lotions, and saline solutions. It is also used in smoke machines to make artificial smoke. As a solvent for food colors and flavorings

Dipropylene glycol ${ }^{9,10}$ finds many uses as a plasticizer, an intermediate in industrial chemical reactions, and as a solvent. Its low toxicity and solvent properties make it an ideal additive for perfumes and skin and hair care products. It is also a common ingredient in commercial fog fluid, used in entertainment industry smoke and haze machines.

Tripropylene Glycol is a viscous, colorless liquid featuring low toxicity, low volatility, slow evaporation and water miscibility. It and its ethers are not toxic and can be used in food, soaps and personal care products as well as industrial.

Commercially available glycol based fog fluids are safe but may be costly and not widely available in our country. So locally manufactured Fog juice is used in our entertainment industry. Most fog juice manufacturers present their fluid as top secret formulas. Most local manufacturers prepare their fluid by their won way. We know there are numerous kinds of glycols and most of them are poisonous. Using the wrong chemical or in wrong concentration may make the fluid toxic. Illiteracy, ignorance and tendency to have more profit, may lead to the production fog fluid that is poisonous. Death of the above mentioned patient was due to the drinking of such a toxic fluid. We collected the fluid and sent it to Bangladesh Council of Scientific \& Industrial Research BCSIR (Science laboratory) for its chemical analysis. But the laboratory failed to analyze it and to give it's chemical composition. The physical appearance of the acquiesced fluid and the mode of death of the patient suggest that it contains substantial amount of DEG. Similar deaths from ARF following accidental drinking of fog fluid have also been noticed by other observers at other parts of the country.

\section{Conclusion:}

Diethylene glycol poisoning is not unknown to us. Between 1990 and 1992, 339 children developed kidney failure, and most of them died, after being given paracetamol (acetaminophen) syrup contaminated with diethylene glycol. ${ }^{11}$ In 2009 similarly 29 deaths were also reported from Brahman Baria district due to DEG in Syrup Paracetamol. Fog juice poisoning is a new addition to the older issue. The physicians have to become alert about its occurrence, so that appropriate and timely intervention can save lives. It is also time to take measures to create public awareness to prevent such unwanted deaths. Nevertheless we have only noticed the fatal effect of the poison after ingestion but we don't know its effect on the performers and other people 
working on the stage who are inhaling the poisonous gas chronically.

\section{Conflict of Interest : None}

\section{References:}

1. Siegfried Rebsdat1 and Dieter Mayer "Ethylene Glycol" in Ullmann's Encyclopedia of Industrial Chemistry, 2002, Wiley-VCH, Weinheim.doi:10.1002/14356007.a10_101.

2. Safety Officer in Physical Chemistry (November 23, 2009). "Safety (MSDS) data for ethylene glycol". Oxford University. http://msds.chem.ox.ac.uk/ET/ethylene_ glycol.html. Retrieved December 30, 2009.

3. Ethylene glycol. National Institute for Occupational Safety and Health. Emergency Response Database. August 22, 2008. Retrieved December 31, 2008.

4. Schep LJ, Slaughter RJ, Temple WA, Beasley DM (2009). "Diethylene glycol poisoning". Clin Toxicol (Phila) 47 (6): 525-35.

5. Robertson OH (1949). "Disinfection of the air with triethylene glycol vapor". The American Journal of Medicine 7 (3): 293-296.
6. Smolinske, Susan C. (1992). Handbook of Food, Drug, and Cosmetic Excipients. Boca Raton: CRC Press. p. 287. ISBN 084933585X.

7. Nalam PC, Clasoahm JN, Mashaghi A et al. Macrotribological Studies of Poly(L-lysine)-graft-Poly(ethylene glycol) in Aqueous Glycerol Mixtures (2010) http:// www.springerlink.com/content/71283620353453pk/]

8. "Safety Data Sheet Product Corexit EC9527A".http:// www.deepwaterhorizonresponse. com/posted/2931/ Corexit_EC9527A_MSDS.539295.pdf. Retrieved 2010-0516.

9. "Dipropylene Glycol Regular Grade (DPG)". Dow Chemical. http://www.dow.com/propyleneglycol/prod/dpg.htm. Retrieved 2009-04-07.

10. "Dipropylene Glycol LO+ (DPG LO+)". Dow Chemical. http://www.dow.com/propyleneglycol/prod/dpglo.htm. Retrieved 2009-04-07.

11. SchHanif M, Mobarak MR, Ronan A, Rahman D, Donovan JJ Jr, Bennish ML. (1995). "Fatal renal failure caused by diethylene glycol in paracetamol elixir: the Bangladesh epidemic". BMJ 311 (6997): 88-91. 